\title{
EQUATIONS AND SYZYGIES OF THE FIRST SECANT VARIETY TO A SMOOTH CURVE
}

\author{
PETER VERMEIRE
}

(Communicated by Irena Peeva)

\begin{abstract}
We show that if $C$ is a linearly normal smooth curve embedded by a line bundle of degree at least $2 g+3+p$, then the secant variety to the curve satisfies $N_{3, p}$.
\end{abstract}

\section{INTRODUCTION}

We work throughout over an algebraically closed field $k$ of characteristic zero. If $X \subset \mathbb{P}^{n}$ is a smooth variety, then we let $\Sigma_{i}(X)$ (or just $\Sigma_{i}$ if the context is clear) denote the (complete) variety of $(i+1)$-secant $i$-planes. Though secant varieties are a classical subject, the majority of the work done involves determining the dimensions of secant varieties to well-known varieties. Perhaps the two most well-known results in this direction are the solution by Alexander and Hirschowitz (completed in [1]) of the Waring problem for homogeneous polynomials and the classification of the Severi varieties by Zak [16.

More recently there has been great interest, e.g. related to algebraic statistics and algebraic complexity, in determining the equations defining secant varieties (e.g. 2 2, 44, 5], 11, [14, 19, 21]). In this work, we use the detailed geometric information concerning secant varieties developed by Bertram [3], Thaddeus 22], and the author [23] to study not just the equations defining secant varieties, but the syzygies among those equations as well.

It was conjectured in [8] and it was shown in [18 that if $C$ is a smooth curve embedded by a line bundle of degree at least $4 g+2 k+3$, then $\Sigma_{k}$ is set theoretically defined by the $(k+2) \times(k+2)$ minors of a matrix of linear forms. This was extended in [12], where the degree bound was improved to $4 g+2 k+2$ and it was shown that the secant varieties are scheme theoretically cut out by the minors. It was further shown in 24] that if $X \subset \mathbb{P}^{n}$ satisfies condition $N_{2}$, then $\Sigma_{1}\left(v_{d}(X)\right)$ is set theoretically defined by cubics for $d \geq 2$.

In [25] it was shown that if $C$ is a smooth curve embedded by a line bundle of degree at least $2 g+3$, then $\mathcal{I}_{\Sigma_{1}}$ is 5 -regular, and under the same hypothesis it was shown in [20] that $\Sigma_{1}$ is arithmetically Cohen-Macaulay. Together with the analogous well-known facts for the curve $C$ itself ([10, [13], [17]), this led to the following conjecture, extending the one found in 24 .

Received by the editors March 8, 2010.

2010 Mathematics Subject Classification. Primary 14N05, 14H99, 13D02.

(c) 2011 American Mathematical Society Reverts to public domain 28 years from publication 
Conjecture $1.1([20])$. Suppose that $C \subset \mathbb{P}^{n}$ is a smooth linearly normal curve of degree $d \geq 2 g+2 k+1+p$, where $p, k \geq 0$. Then:

(1) $\Sigma_{k}$ is $A C M$ and $\mathcal{I}_{\Sigma_{k}}$ has regularity $2 k+3$ unless $g=0$, in which case the regularity is $k+2$.

(2) $\beta_{n-2 k-1, n+1}\left(\Sigma_{k}\right)=\left(\begin{array}{l}g+k \\ k+1\end{array}\right)$.

(3) $\Sigma_{k}$ satisfies $N_{k+2, p}$.

Remark 1.2. Recall [7] that a variety $Z \subset \mathbb{P}^{n}$ satisfies $\mathrm{N}_{r, p}$ if the ideal of $Z$ is generated in degree $r$ and the syzygies among the equations are linear for $p-1$ steps. Note that the better-known condition $N_{p}$ [13] implies $N_{2, p}$.

By the work of Green and Lazarsfeld [13, [15, the conjecture holds for $k=0$. Further, by [9] and by [27] it holds for $g \leq 1$, and by [20] parts (1) and (2) hold for $k=1$. In this work, we show that part (3) holds for $k=1$ (Theorem 3.5). Some analogous results for higher dimensional varieties can be found in [26].

Our approach combines the geometric knowledge of secant varieties mentioned above with the well-known Koszul approach of Green and Lazarsfeld. To fix notation, if $L$ is a vector bundle on a smooth curve $C$, then we let $\mathcal{E}_{L}=d_{*}(L \otimes \mathcal{O})$, where $d: C \times C \rightarrow S^{2} C$ is the natural double cover, and if $\mathcal{F}$ is a globally generated coherent sheaf on a variety $X$, then we have the coherent sheaf $M_{\mathcal{F}}$ defined via the exact sequence $0 \rightarrow M_{\mathcal{F}} \rightarrow \Gamma(X, \mathcal{F}) \rightarrow \mathcal{F} \rightarrow 0$. As we will be interested only in the first secant variety for the remainder of the paper, we write $\Sigma$ for $\Sigma_{1}$.

\section{Preliminaries}

Our starting point is the familiar:

Proposition 2.1. Let $C \subset \mathbb{P}^{n}$ be a smooth curve embedded by a line bundle $L$ of degree at least $2 g+3$. Then $\Sigma$ satisfies $\mathrm{N}_{3, p}$ if and only if $H^{1}\left(\Sigma, \wedge^{a} M_{L}(b)\right)=0$, $2 \leq a \leq p+1, b \geq 2$.

Proof. Because $L$ also induces an embedding $\Sigma \subset \mathbb{P}^{n}$, we abuse notation and denote the associated vector bundle on $\Sigma$ by $M_{L}$. Letting $F=\bigoplus \Gamma\left(\Sigma, \mathcal{O}_{\Sigma}(n)\right)$ and applying [6, 5.8] to $\mathcal{O}_{\Sigma}$ give the exact sequence:

$$
0 \rightarrow \operatorname{Tor}_{a-1}(F, k)_{a+b} \rightarrow H^{1}\left(\Sigma, \bigwedge^{a} M_{L}(b)\right) \rightarrow H^{1}\left(\Sigma, \bigwedge^{a} \Gamma(\mathcal{O}(1)) \otimes \mathcal{O}_{\Sigma}(b)\right) .
$$

As $\Sigma$ is ACM [20], the term on the right vanishes.

Notation and Terminology 2.2. Under the hypothesis that $\operatorname{deg}(L) \geq 2 g+3$, the reader should keep in mind the following morphisms [23]:

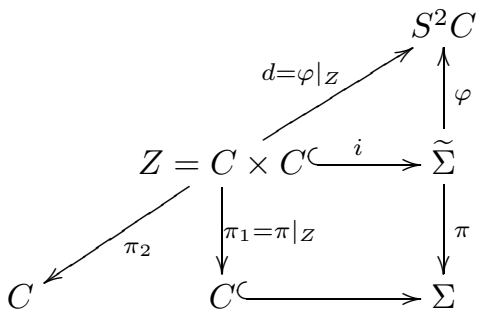

where

- $\pi$ is the blow up of $\Sigma$ along $C$.

- $i$ is the inclusion of the exceptional divisor of the blow-up. 
- $d$ is the double cover; $\pi_{i}$ are the projections.

- $\varphi$ is the morphism induced by the linear system $|2 H-E|$ which gives $\widetilde{\Sigma}$ the structure of a $\mathbb{P}^{1}$-bundle over $S^{2} C$; note in particular that $\widetilde{\Sigma}$ is smooth.

We make frequent use of the rank 2 vector bundle $\mathcal{E}_{L}=\varphi_{*} \mathcal{O}(H)=d_{*}(L \otimes \mathcal{O})$ and the fact [25, Proposition 9] that $R^{i} \pi_{*} \mathcal{O}_{\widetilde{\Sigma}}=H^{i}\left(C, \mathcal{O}_{C}\right) \otimes \mathcal{O}_{C}$ for $i \geq 1$.

Proposition 2.3. If $C$ is a smooth curve embedded by a line bundle $L$ with $\operatorname{deg}(L) \geq$ $2 g+3$, then $\Sigma$ satisfies $\mathrm{N}_{3, p}$ if and only if

$$
H^{1}\left(\widetilde{\Sigma}, \pi^{*} \bigwedge^{a} M_{L}(b)\right) \rightarrow H^{0}\left(\Sigma, \bigwedge^{a} M_{L}(b) \otimes R^{1} \pi_{*} \mathcal{O}_{\widetilde{\Sigma}}\right)
$$

is injective for $2 \leq a \leq p+1, b \geq 2$.

Proof. This follows immediately from the 5 -term sequence associated to the LeraySerre spectral sequence,

$$
0 \rightarrow H^{1}\left(\Sigma, \bigwedge^{a} M_{L}(b)\right) \rightarrow H^{1}\left(\widetilde{\Sigma}, \pi^{*} \bigwedge^{a} M_{L}(b)\right) \rightarrow H^{0}\left(\Sigma, \bigwedge^{a} M_{L}(b) \otimes R^{1} \pi_{*} \mathcal{O}_{\widetilde{\Sigma}}\right),
$$

and Proposition 2.1.

We will need a cohomological result:

Lemma 2.4. Let $C \subset \mathbb{P}^{n}$ be a smooth curve embedded by a line bundle $L$ with $\operatorname{deg}(L) \geq 2 g+3$. Then $H^{i}\left(\widetilde{\Sigma}, \mathcal{O}_{\widetilde{\Sigma}}(b H-E)\right)=0$ for $i, b \geq 1$.

Proof. Because $C$ is projectively normal we have $H^{i}\left(\widetilde{\mathbb{P}}^{n}, \mathcal{O}_{\widetilde{\mathbb{P}} n}(b H-E)\right)=0$ for $i, b \geq 1$. Thus $H^{i}\left(\widetilde{\Sigma}, \mathcal{O}_{\widetilde{\Sigma}}(b H-E)\right)=H^{i+1}\left(\widetilde{\mathbb{P}}^{n}, \mathcal{O}_{\widetilde{\mathbb{P}} n}(b H-E) \otimes \mathcal{I}_{\widetilde{\Sigma}}\right)$, but by $[20$, $2.4(6)]$, we know that $H^{i+1}\left(\widetilde{\mathbb{P}}^{n}, \mathcal{O}_{\widetilde{\mathbb{P}}^{n}}(b H-E) \otimes \mathcal{I}_{\widetilde{\Sigma}}\right)=H^{i+1}\left(\mathbb{P}^{n}, \mathcal{I}_{\Sigma}(b)\right)=0$ for $i \geq 0, b \in \mathbb{Z}$.

\section{MAIN RESUlT}

We first reinterpret the injection in Proposition 2.3 as a cohomological vanishing statement on $\widetilde{\Sigma}$ (Proposition 3.1) , then on $S^{2} C$ (Corollary 3.3), and finally on $C \times C$ (Theorem 3.5).

Proposition 3.1. Let $C \subset \mathbb{P}^{n}$ be a smooth curve satisfying $N_{p}$ embedded by a line bundle $L$ with $\operatorname{deg}(L) \geq 2 g+3$. Then $\Sigma$ satisfies $\mathrm{N}_{3, p}$ if

$$
H^{i}\left(\widetilde{\Sigma}, \pi^{*} \bigwedge^{a-1+i} M_{L} \otimes \mathcal{O}(2 H-E)\right)=0
$$

for $2 \leq a \leq p+1, i \geq 1$.

Proof. We use Proposition 2.3. Consider the sequence on $\widetilde{\Sigma}$,

$$
0 \rightarrow \pi^{*} \bigwedge^{a} M_{L}(b H-E) \rightarrow \pi^{*} \bigwedge^{a} M_{L}(b H) \rightarrow \pi^{*} \bigwedge^{a} M_{L}(b H) \otimes \mathcal{O}_{Z} \rightarrow 0 .
$$

We know that

$$
\begin{aligned}
H^{1}\left(Z, \pi^{*} \bigwedge^{a} M_{L}(b H) \otimes \mathcal{O}_{Z}\right) & =H^{1}\left(Z,\left(\bigwedge^{a} M_{L} \otimes L^{b}\right) \otimes \mathcal{O}_{C}\right) \\
& =H^{1}\left(C, \mathcal{O}_{C}\right) \otimes H^{0}\left(C, \bigwedge^{a} M_{L} \otimes L^{b}\right) \\
& =H^{0}\left(\Sigma, \bigwedge^{a} M_{L}(b) \otimes R^{1} \pi_{*} \mathcal{O}_{\widetilde{\Sigma}}\right)
\end{aligned}
$$


The first equality follows as the restriction of $\pi^{*} \bigwedge^{a} M_{L}(b H)$ to $Z$ is $\bigwedge^{a} M_{L}(b H) \otimes$ $\mathcal{O}_{C}$. For the second we use the Künneth formula together with the fact that $h^{1}\left(C, \bigwedge^{a} M_{L} \otimes L^{b}\right)=0$ as $C$ satisfies $N_{p}[13$. The third is the last part of subsection 2.2 .

Thus

$$
\begin{aligned}
& h^{1}\left(\Sigma, \bigwedge^{a} M_{L}(b)\right)=\operatorname{Rank}\left(H^{1}\left(\widetilde{\Sigma}, \pi^{*} \bigwedge^{a} M_{L}(b H-E)\right)\right. \\
& \left.\rightarrow H^{1}\left(\widetilde{\Sigma}, \pi^{*} \bigwedge^{a} M_{L}(b H)\right)\right),
\end{aligned}
$$

and so by Proposition 2.3 it is enough to show that $H^{1}\left(\widetilde{\Sigma}, \pi^{*} \bigwedge^{a} M_{L} \otimes \mathcal{O}(b H-E)\right)=$ 0 for $2 \leq a \leq p+1, b \geq 2$.

From the sequence

$$
\begin{aligned}
0 & \rightarrow \pi^{*} \bigwedge_{a}^{a+1} M_{L} \otimes \mathcal{O}(b H-E) \rightarrow \bigwedge^{a+1} \Gamma \otimes \mathcal{O}(b H-E) \\
& \rightarrow \pi^{*} \bigwedge M_{L} \otimes \mathcal{O}((b+1) H-E) \rightarrow 0
\end{aligned}
$$

and the fact (Lemma 2.4) that $H^{i}(\widetilde{\Sigma}, \mathcal{O}(b H-E))=0$, we see that

$$
H^{1}\left(\widetilde{\Sigma}, \pi^{*} \bigwedge^{a} M_{L} \otimes \mathcal{O}(b H-E)\right)=H^{b-2}\left(\widetilde{\Sigma}, \pi^{*} \bigwedge^{a+b-2} M_{L} \otimes \mathcal{O}(2 H-E)\right)
$$

for $b \geq 2$.

Lemma 3.2. Let $C \subset \mathbb{P}^{n}$ be a smooth curve embedded by a line bundle $L$ with $\operatorname{deg}(L) \geq 2 g+3$ and consider the morphism $\varphi: \widetilde{\Sigma} \rightarrow S^{2} C \subset \mathbb{P}^{s}$ induced by the linear system $|2 H-E|$. Then $\varphi_{*} \bigwedge^{a} M_{L}=\bigwedge^{a} M_{\mathcal{E}_{\mathcal{L}}}$, and hence

$$
H^{i}\left(\widetilde{\Sigma}, \pi^{*} \bigwedge^{a} M_{L} \otimes \mathcal{O}(2 H-E)\right)=H^{i}\left(S^{2} C, \bigwedge^{a} M_{\mathcal{E}_{L}} \otimes \mathcal{O}_{S^{2} C}(1)\right) .
$$

Proof. Consider the diagram on $\widetilde{\Sigma}$ :

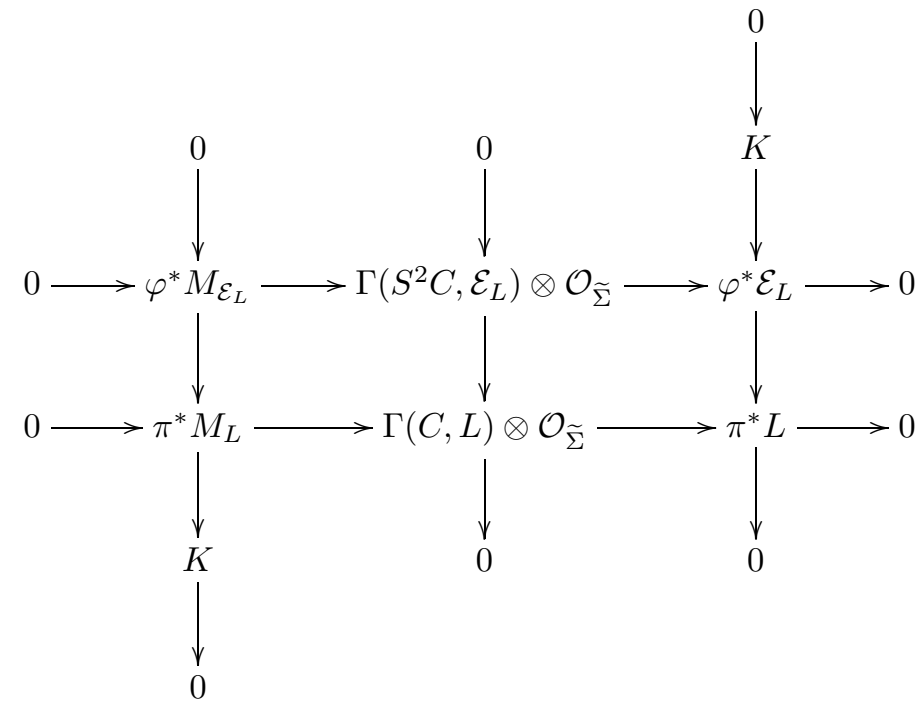


The vertical map in the middle is surjective, as we have $\Gamma\left(S^{2} C, \mathcal{E}_{L}\right)=\Gamma(\widetilde{\Sigma}, \mathcal{O}(H))=$ $\Gamma(C \times C, L \otimes \mathcal{O})=\Gamma(C, L)$. Therefore, surjectivity of the lower right horizontal map and commutativity of the diagram show that the right-hand vertical map is surjective.

Note that $R^{i} \varphi_{*} \varphi^{*} \mathcal{E}_{L}=\mathcal{E}_{L} \otimes R^{i} \varphi_{*} \mathcal{O}_{\widetilde{\Sigma}}$ by the projection formula and that the higher direct image sheaves $R^{i} \varphi_{*} \mathcal{O}_{\widetilde{\Sigma}}$ vanish as $\widetilde{\Sigma}$ is a $\mathbb{P}^{1}$-bundle over $S^{2} C$. For the higher direct images, we have $R^{i} \varphi_{*} \pi^{*} L=0$ as the restriction of $L$ to a fiber of $\varphi$ is $\mathcal{O}(1)$, and hence the cohomology along the fibers vanishes. From the rightmost column, we see that $R^{i} \varphi_{*} K=0$. From the leftmost column, we have the sequence

$$
0 \rightarrow \varphi^{*} \bigwedge^{a} M_{\mathcal{E}_{L}} \rightarrow \pi^{*} \bigwedge^{a} M_{L} \rightarrow \varphi^{*} \bigwedge^{a-1} M_{\mathcal{E}_{L}} \otimes K \rightarrow 0,
$$

but as $R^{i} \varphi_{*}\left(K \otimes \varphi^{*} \bigwedge^{a-1} M_{\mathcal{E}_{L}}\right)=R^{i} \varphi_{*} K \otimes \bigwedge^{a-1} M_{\mathcal{E}_{L}}=0$, we have $\varphi_{*} \bigwedge^{a} M_{L}=$ $\bigwedge^{a} M_{\mathcal{E}_{L}}$.

Combining Proposition 3.1 with Lemma 3.2 yields:

Corollary 3.3. Let $C \subset \mathbb{P}^{n}$ be a smooth curve satisfying $N_{p}$ embedded by a line bundle $L$ with $\operatorname{deg}(L) \geq 2 g+3$. Then $\Sigma$ satisfies $\mathrm{N}_{3, p}$ if

$$
H^{i}\left(S^{2} C, \bigwedge^{a-1+i} M_{\mathcal{E}_{L}} \otimes \mathcal{O}(1)\right)=0
$$

for $2 \leq a \leq p+1, i \geq 1$.

We need a technical lemma, completely analogous to [15, 1.4.1].

Lemma 3.4. Let $X \subset \mathbb{P}^{n}$ be a smooth curve embedded by a non-special line bundle $L$ satisfying $N_{2,2}$, let $x_{1}, \cdots, x_{n-2}$ be a general collection of distinct points, and let $D=x_{1}+\cdots+x_{n-2}$. Then there is an exact sequence of vector bundles on $X \times X$,

$$
0 \rightarrow L^{-1}(D) \otimes L^{-1}(D)(\Delta) \rightarrow d^{*} M_{\mathcal{E}_{L}} \rightarrow \bigoplus_{i}\left(\mathcal{O}\left(-x_{i}\right) \otimes \mathcal{O}\left(-x_{i}\right)\right) \rightarrow 0 .
$$

Proof. Choose a general point $x_{1} \in X$ and consider the following diagram on $X \times X$ :

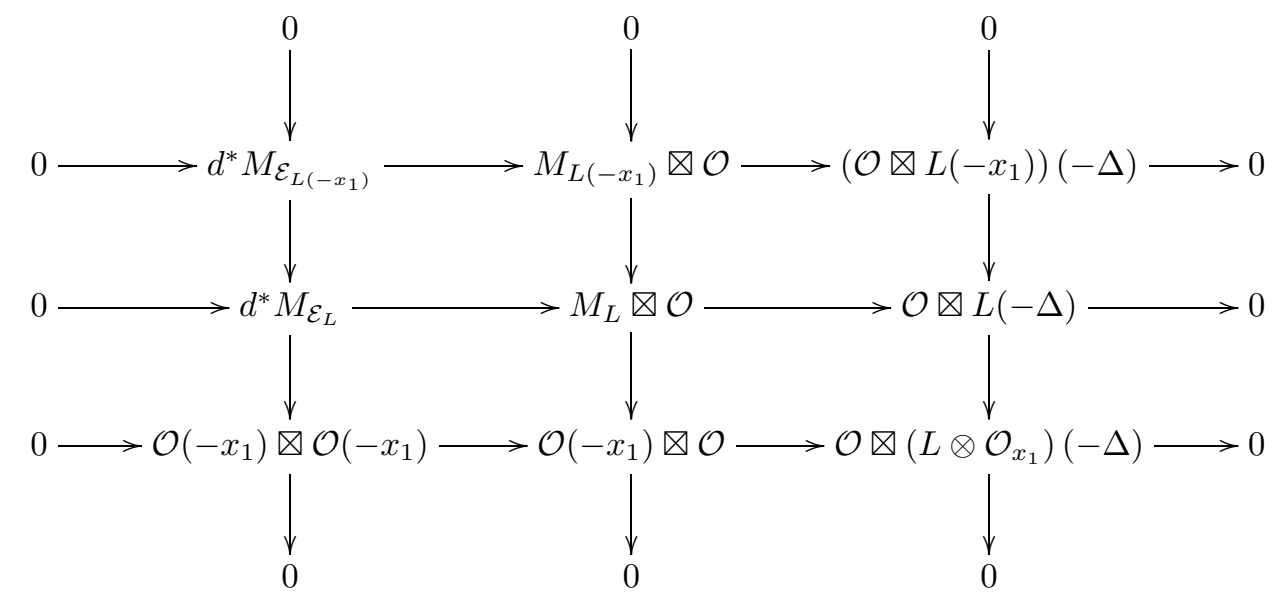


where the center column comes from [15, 1.4.1]. Following just as in that proof, we obtain

$$
0 \rightarrow d^{*} M_{\mathcal{E}_{L(-D)}} \rightarrow d^{*} M_{\mathcal{E}_{L}} \rightarrow \bigoplus_{i}\left(\mathcal{O}\left(-x_{i}\right) \otimes \mathcal{O}\left(-x_{i}\right)\right) \rightarrow 0
$$

from the left column. Note however that $d^{*} M_{\mathcal{E}_{L(-D)}}$ is a line bundle, and one checks that $d^{*} M_{\mathcal{E}_{L(-D)}}=\bigwedge^{2} \mathcal{E}_{L(-D)}^{*}=L^{-1}(D) \otimes L^{-1}(D)(\Delta)$.

Theorem 3.5. Let $C \subset \mathbb{P}^{n}$ be a smooth curve embedded by a line bundle $L$ with $\operatorname{deg}(L) \geq 2 g+p+3, p \geq 0$. Then $\Sigma$ satisfies $\mathrm{N}_{3, p}$.

Proof. First note [13] that such a curve satisfies $N_{p+2}$. We verify the condition in Corollary 3.3. Pulling the sequence on $S^{2} C$,

$$
0 \rightarrow M_{\mathcal{E}_{L}} \rightarrow \Gamma\left(S^{2} C, \mathcal{E}_{L}\right) \rightarrow \mathcal{E}_{L} \rightarrow 0,
$$

back to $Z=C \times C$ yields the diagram

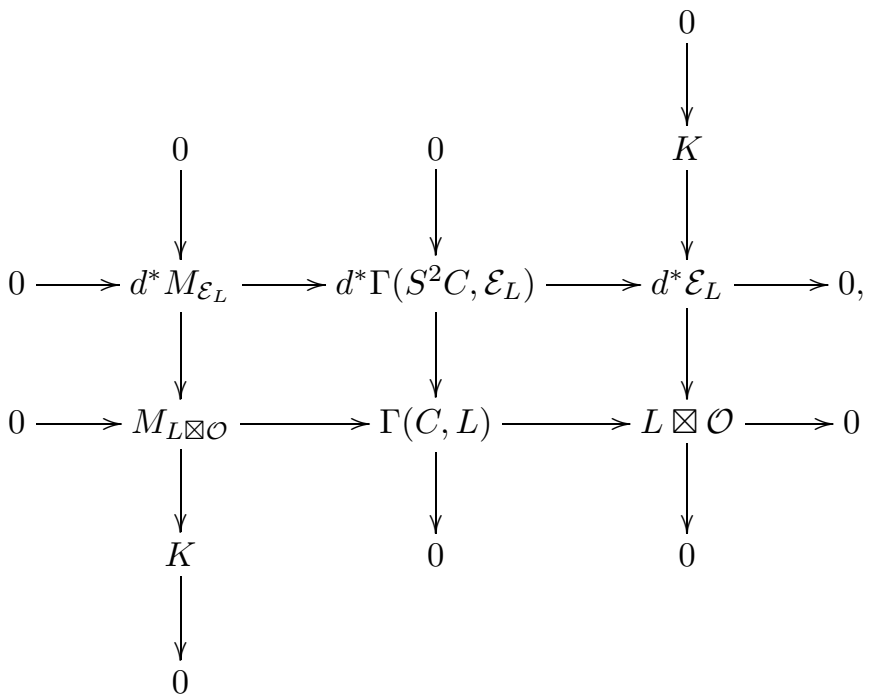

As in 20] we have $d_{*} \mathcal{O}_{Z}=\mathcal{O}_{S^{2} C} \oplus M$ where $d^{*} M=\mathcal{O}(-\Delta), d_{*} K=\mathcal{E}_{L} \otimes M$, and $K=d^{*} \bigwedge^{2} \mathcal{E}_{L} \otimes\left(L^{*} \otimes \mathcal{O}\right)=\mathcal{O} \otimes L(-\Delta)$. From the left vertical sequence we have

$$
0 \rightarrow \bigwedge^{a} d^{*} M_{\mathcal{E}_{L}} \rightarrow \bigwedge^{a} M_{L \otimes \mathcal{O}} \rightarrow \bigwedge^{a-1} d^{*} M_{\mathcal{E}_{L}} \otimes K \rightarrow 0,
$$

and pushing down to $S^{2} C$ yields

$$
\begin{aligned}
0 & \rightarrow \bigwedge^{a} M_{\mathcal{E}_{L}} \oplus\left(\bigwedge^{a} M_{\mathcal{E}_{L}} \otimes M\right) \rightarrow d_{*} \bigwedge^{a} M_{L \otimes \mathcal{O}} \\
& \rightarrow \bigwedge^{a-1} M_{\mathcal{E}_{L}} \otimes \mathcal{E}_{L} \otimes M \rightarrow 0 .
\end{aligned}
$$

Twisting this sequence by $\mathcal{O}_{S^{2} C}(1) \otimes M^{*}$ gives

$$
\begin{aligned}
0 & \rightarrow \bigwedge^{a} M_{\mathcal{E}_{L}}(1) \otimes M^{*} \oplus \bigwedge^{a} M_{\mathcal{E}_{L}}(1) \mathcal{O}_{S^{2} C}(1) \otimes M^{*} \otimes d_{*} \bigwedge^{a} M_{L \otimes \mathcal{O}} \\
& \rightarrow \bigwedge^{a-1} M_{\mathcal{E}_{L}}(1) \otimes \mathcal{E}_{L} \rightarrow 0
\end{aligned}
$$


Since $d^{*} \mathcal{O}_{\operatorname{Hilb}^{2} X}(1) \otimes M^{*}=L \otimes L \otimes \mathcal{O}(-\Delta)$, it suffices to show that

$$
H^{i}\left(Z, \bigwedge^{a-1+i} d^{*} M_{\mathcal{E}_{L}} \otimes L \otimes L \otimes \mathcal{O}(-\Delta)\right)=0
$$

for $2 \leq a \leq p+1, i=1,2$.

Now, by Lemma 3.4 we have exact sequences

$$
\begin{aligned}
0 & \rightarrow \bigwedge_{r}^{r-1} Q \otimes \mathcal{O}(D) \otimes \mathcal{O}(D) \rightarrow \bigwedge^{r} d^{*} M_{\mathcal{E}_{L}} \otimes L \otimes L \otimes \mathcal{O}(-\Delta) \\
& \rightarrow \bigwedge^{r} Q \otimes L \otimes L \otimes \mathcal{O}(-\Delta) \rightarrow 0,
\end{aligned}
$$

where $Q=\bigoplus_{i}\left(\mathcal{O}\left(-x_{i}\right) \otimes \mathcal{O}\left(-x_{i}\right)\right)$.

On the right, we have a direct sum of vector bundles of the form $F \otimes F(-\Delta)$, where $F$ is a line bundle of degree $\operatorname{deg}(L)-r$. Thus $H^{1}$ and $H^{2}$ of the right side will vanish when $\operatorname{deg}(L)-r \geq 2 g+1$.

On the left, we have a direct sum of vector bundles of the form $F \otimes F$, where $F$ is a line bundle of degree $n-2-(r-1)=\operatorname{deg}(L)-g-r-1$. Because $x_{1}, \cdots, x_{n-2}$ are general, $H^{1}$ and $H^{2}$ of the left side will vanish when $\operatorname{deg}(L)-g-r-1 \geq g$. Combining these, we see that $H^{i}\left(Z, \bigwedge^{a-1+i} d^{*} M_{\mathcal{E}_{L}} \otimes L \otimes L \otimes \mathcal{O}\left(-E_{\Delta}\right)\right)=0$ for $2 \leq a \leq p+1, i=1,2$, as long as $\operatorname{deg}(L) \geq 2 g+p+3$.

\section{ACKNOWLEDGMENT}

This project grew out of work done together with Jessica Sidman and has benefited greatly from her insight and input, as well as from her comments regarding preliminary drafts of this work.

\section{RefERENCES}

[1] J. Alexander and A. Hirschowitz, Polynomial interpolation in several variables, J. Algebraic Geom. 4 (1995), no. 2, 201-222. MR1311347 (96f:14065)

[2] E. S. Allman and J. A. Rhodes, Phylogenetic ideals and varieties for the general Markov model, Adv. in Appl. Math. 40 (2008), no. 2, 127-148. MR.2388607 (2008m:60145)

[3] A. Bertram, Moduli of rank-2 vector bundles, theta divisors, and the geometry of curves in projective space, J. Diff. Geom. 35 (1992), 429-469. MR1158344 (93g:14037)

[4] M. V. Catalisano, A. V. Geramita, and A. Gimigliano, On the ideals of secant varieties to certain rational varieties, J. Algebra 319 (2008), no. 5, 1913-1931. MR2392585 (2009g:14068)

[5] D. Cox and J. Sidman, Secant varieties of toric varieties, J. Pure Appl. Algebra 209 (2007), no. 3, 651-669. MR2298847 (2008i:14077)

[6] D. Eisenbud, The Geometry of Syzygies, Springer-Verlag, New York, 2005. MR2103875 (2005h:13021)

[7] D. Eisenbud, M. Green, K. Hulek, and S. Popescu, Restricting linear syzygies: Algebra and geometry, Compos. Math. 141 (2005), 1460-1478. MR2188445 (2006m:14072)

[8] D. Eisenbud, J. Koh, and M. Stillman, Determinantal equations for curves of high degree, Amer. J. Math. 110 (1988), no. 3, 513-539. MR944326 (89g:14023)

[9] T. Fisher, The higher secant varieties of an elliptic normal curve, preprint.

[10] T. Fujita, Defining equations for certain types of polarized varieties. Complex analysis and algebraic geometry, 165-173. Iwanami Shoten, Tokyo, 1977. MR0437533 (55:10457)

[11] L. Garcia, M. Stillman, and B. Sturmfels, Algebraic geometry of Bayesian networks, J. Symbolic Comput. 39 (2005), no. 3-4, 331-355. MR2168286 (2006g:68242)

[12] A. Ginensky, A generalization of the Clifford index and determinental equations for curves and their secant varieties, $\mathrm{PhD}$ thesis, University of Chicago, 2008. MR2712616

[13] M. Green, Koszul cohomology and the geometry of projective varieties, J. Diff. Geom. 19 (1984), 125-171. MR739785(85e:14022) 
[14] J. M. Landsberg and J. Weyman, On secant varieties of compact Hermitian symmetric spaces, J. Pure Appl. Algebra 213 (2009), no. 11, 2075-2086. MR2533306 (2010i:14095)

[15] R. Lazarsfeld, A sampling of vector bundle techniques in the study of linear series, in Lectures on Riemann surfaces (Trieste, 1987), 500-559, World Sci. Publ., Teaneck, NJ, 1989. MR 1082360 (92f:14006)

[16] R. Lazarsfeld and A. Van de Ven, Topics in the geometry of projective space. Recent work of F. L. Zak. With an addendum by Zak. DMV Seminar, 4. Birkhäuser Verlag, Basel, 1984. MR808175 (87e:14045)

[17] D. Mumford, Varieties defined by quadratic equations. 1970 Questions on Algebraic Varieties (C.I.M.E., III Ciclo, Varenna, 1969), 29-100, Edizioni Cremonese, Rome. MR0282975 (44:209)

[18] M.S. Ravi, Determinantal equations for secant varieties of curves, Comm. Algebra 22 (1994), no. 8, 3103-3106. MR 1272376 (95c:14029)

[19] J. Sidman and S. Sullivant, Prolongations and computational algebra, Canad. J. Math. 61 (4) (2009), 930-949. MR2541390 (2010h:14084)

[20] J. Sidman and P. Vermeire, Syzygies of the secant variety of a curve, Algebra and Number Theory 3 (2009), no. 4, 445-465. MR.2525559(2010j:13026)

[21] B. Sturmfels and S. Sullivant, Combinatorial secant varieties, Pure Appl. Math. Q. 2 (2006), no. 3, 867-891. MR2252121 (2007h:14082)

[22] M. Thaddeus, Stable pairs, linear systems and the Verlinde formula, Invent. Math. 117 (1994), no. 2, 317-353. MR1273268 (95e:14006)

[23] P. Vermeire, Some results on secant varieties leading to a geometric flip construction, Compos. Math. 125 (2001), no. 3, 263-282. MR1818982 (2002c:14027)

[24] P. Vermeire, Secant varieties and birational geometry, Math. Z. 242 (2002), no. 1, 75-95. MR.1985451 (2004e:14025)

[25] P. Vermeire, Regularity and normality of the secant variety to a projective curve, J. Algebra 319 (2008), no. 3, 1264-1270. MR2379097 (2009b:14099)

[26] P. Vermeire, Arithmetic properties of the secant variety to a projective variety, preprint.

[27] H.-C. Graf v. Bothmer and K. Hulek, Geometric syzygies of elliptic normal curves and their secant varieties, Manuscripta Math. 113 (2004), 35-68. MR.2135560 (2006b:14009)

Department of Mathematics, 214 Pearce Hall, Central Michigan University, Mount Pleasant, Michigan 48859

E-mail address: p.vermeire@cmich.edu 